\title{
EDITORIAL
}

\section{Eosinophilic bronchiolitis: is it a new syndrome?}

\author{
Venerino Poletti
}

( dentification of new diseases or a cluster (syndrome) of disorders with very similar pathological, radiological and clinical aspects, even sharing common pathogenic steps, is an important fact to build up the scientific soul of the complex and intriguing art called medicine. In this issue of the European Respiratory Journal, a new distinct syndrome, hypereosinophilic obliterative bronchiolitis, is described by CORDIER et al. [1]. This syndrome is defined by: 1) a blood eosinophil cell count $>1 \mathrm{G} \cdot \mathrm{L}^{-1}$ and/or a bronchoalveolar lavage (BAL) eosinophil differential cell count $>25 \%$; 2) persistent airflow obstruction on lung function tests not modifiable after 4-6 weeks of inhaled corticosteroid therapy $\left(2000 \mu \mathrm{g} \cdot \mathrm{day}^{-1}\right.$ of beclometasone or equivalent); and 3) a lung biopsy showing inflammatory bronchiolitis with prominent bronchiolar wall infiltration by eosinophils and/or characteristic direct high-resolution computed tomography (HRCT) features of bronchiolitis (poorly defined centrilobular nodules, branching opacities and tree-inbud pattern). The first impression that a cursory clinician and/ or pathologist may have, going swiftly through this list of criteria, is that "we are dealing with a severe and persistent form of asthma". In chronic asthma the obstructive impairment becomes fixed and not modifiable, at least not easily, with steroids or bronchodilators. In chronic asthma eosinophils in lung tissue and blood may be constantly increased and HRCT scans show abnormalities in between $68 \%$ and $90 \%$ of patients (bronchial wall thickening and narrowing of bronchial lumen, cylindric bronchiectasis, thick linear opacities, areas of decreased attenuation, bronchial mucoid impaction, small centrilobular opacities, airspace consolidation and thin-walled cysts) [2]. Finally, from a pathological point of view, mucous eosinophilic plugs may fill both small bronchi and bronchioles, and marked goblet cell hyperplasia/metaplasia of bronchial/ bronchiolar epithelium, thickening of the basement membrane, bronchiolar smooth muscle hypertrophy, fibrosis of the bronchiolar wall with peribronchiolar lymphoid aggregates, and mixed inflammatory infiltrate with prominent eosinophils throughout airway walls are all detectable [3]. However, it is clear that the concept of "severe and persistent asthma" is still poorly understood and the article by CORDIER et al. [1] may help to better define and appreciate the characteristics (clinical, radiographic, pathologic and biologic) of those patients that have symptoms, signs, imaging findings and even pathologic aspects as boundaries between severe asthma and some form of a not yet well-defined bronchiolitis.

Dept of Diseases of the Thorax, Ospedale G.B. Morgagni, Forli, Italy.

CORRESPONDENCE: V. Poletti, Ospedale G.B. Morgagni-L. Pierantoni, U.O. Pneumologia, Via Forlanini 34, Forli, Italy. E-mail: venerino.poletti@gmail.com
In the study by CORDIER et al.[1], one patient had a limited form of Churg-Strauss syndrome. One patient showed an aberrant population of T-lymphocytes in the peripheral blood (CD3+ CD4+CD7- lymphocytes) with oligoclonal T-cell receptor gamma VG9J1J2 re-arrangement suggesting a relationship with the lymphocytic variant of hypereosinophilic syndrome [4]. One patient had the typical aspects of allergic bronchopulmonary mycosis (as Aspergillus was not documented either by immunological tests, microbiology or histology, a diagnosis of allergic bronchopulmonary aspergillosis may not be accepted) [3]; and, finally, in one patient (not included here but part of the clinical experience of the authors), the lesion was allegedly related to minocycline use. Almost all the patients had airflow obstruction despite inhaled therapy. HRCT features indicated that the lesions were centered on small bronchi/bronchioles (bronchial wall thickening, tree-inbud pattern, mucoid impaction, finger-in-glove pattern and centrilobular nodules), and the burden of eosinophilic infiltrate in the airway walls was higher than expected in asthma and rather typical of cellular bronchiolitis [5]. Finally, in two patients, bronchoscopy documented patchy tracheobronchial mucosal lesions corresponding histopathologically to ulcerated areas of necrosis and prominent eosinophilic inflammation; paranasal sinus inflammation was well documented in four out of six patients confirming that the lesions also extended to the larger respiratory airways. Improvement was observed after oral steroids or even immunosuppressor drug use.

The box inside which hypereosinohilic obliterative bronchiolitis syndrome might be included, with direct computed tomography (CT) scan findings indicating small airways disease, eosinophilic bronchiolitis and blood/BAL eosinophilia as selective criteria, contains other entities or specific subsets of patients.

In Churg-Strauss syndrome, it is already known that occasionally eosinophilic inflammatory lesions may primarily involve the bronchovascular bundles with minimal spread to the alveolar parenchyma [6], and bronchoscopy may detect lesions even in larger airways. Eosinophilic bronchiolitis was reported by Japanese authors [7], and common characteristics included bronchiolitis with eosinophilic infiltration, CT scan findings similar to that observed in diffuse panbronchiolitis, ventilatory impairment, airway hyperreactivity, and blood and BAL fluid eosinophilia. Recently, Wenzel et al. [8] described 10 patients with clinically severe asthma with subtle CT scan lesions (a tree-in-bud pattern was only detected in one patient) and with characteristic pathologic aspects; peripheral airways showed asthmatic changes (submucosal inflammation consisting of eosinophils, neutrophils, lymphocytic transmigration, 
submucosal fibrosis, mucus plugging, peribronchiolar fibrosis, bronchioloectasis, and muscular hypertrophy along with interstitial mononuclear pneumonia with the presence of poorly formed granulomas, lymphoid aggregates and airspace organisation). WENZEL et al. [8] labelled this subset of patients as "asthmatic granulomatosis". Interestingly, non-steroidal cytotoxic drugs improved the course of the disease. The description and data collected by CORDIER et al. [1], therefore, add some other element of knowledge to this not yet welldefined ensemble: some patients had an autoimmune disorder (Churg-Strauss syndrome), others hypersensitivity behaviour (allergic bronchopulmonary mycosis), some had a disregulation of T-lymphocytes (probably including lymphocytes expressing and releasing interleukin-5), and some had elements indicating a drug reaction.

Therefore, hypereosinophilic obliterative bronchiolitis along with other recently reported "entities" indicate that patients usually considered as affected by severe asthma may actually have a disorder in which different biological processes are involved but which share common elements, including: 1) small airways where the anatomic zone is more heavily involved (although the infiltrate may extend to the large airways and paranasal sinuses); 2) eosinophils being the more represented cells in the morphologic lesion; 3) dysregulation of immunity (auto-antibodies production, hypersensitivity, disregulation of T-cells) may have a role; and 4) non-steroidal immunosuppressant drugs might be effective (confirming the empirical feeling of expert clinicians).

This report could open new windows drawing attention to the links between auto-immunity, hypersensitivity, T-cell dysregulation, eosinophilic inflammation and bronchiolitis. It raises interest in new pathogenetic mechanisms leading to obliterative bronchiolitis, discloses links between oligoclonal T-cell proliferation and bronchiolocentric eosinophilic inflammation, and suggests that cytotoxic drugs or even monoclonal antibodies might have some benefit. The next step to exploit these new scenarios could be the collection of a significant number of patients with these clinical-radiological characteristics provided with pathological (and biological) data. In fact, CORDIER et al. [1] reported histopathological information in only two patients. Interestingly, as the lesions are centrilobular, a bronchoscopic approach (transbronchial lung biopsy with common flexible forceps, jumbo forceps or even cryoprobes) could obviate the need of a surgical approach. In conclusion, the study by CORDIER et al. [1] reminds us that a good clinical reasoning is at the basis of well oriented research in medicine.

\section{STATEMENT OF INTEREST}

None declared.

\section{REFERENCES}

1 Cordier J-F, Cottin V, Khouatra C, et al. Hypereosinophilic obliterative bronchiolitis: a distinct, unrecognised syndrome. Eur Respir J 2013; 41: 1126-1134.

2 Diseases of the airways. In: Hansell DM, Lynch DA, McAdams HP, et al., eds. Imaging of Diseases of the Chest. 5th Edn. London, Mosby, 2010; pp. 752-754.

3 Guinee DG Jr. Asthma and related eosinophilic infiltrates. In: Tomasheski JF Jr, Cagle PT, Farver CF, et al., eds. Dail and Hammar's Pulmonary Pathology. Vol I Non-neoplastic Lung Disease. 3rd Edn. New York, Springer, 2008; pp. 561-599.

4 Bain BJ. Eosinophilia and chronic eosinophilic leukemia, including myeloid/lymphoid neoplasms with eosinophilia and abnormalities of PDGFRA, PDGGRB, and FGFR1. In: Jaffe ES, Lee Harris N, Vardiman JW, et al., eds. Hematopathology. Philadelphia, Saunders, 2010; pp. 777-787.

5 Poletti V, Casoni G, Zompatori M, et al. Bronchiolitis. In: Cordier J-F, ed. Orphan Lung Diseases. Eur Respir Monogr 2011; 54: 84-103.

6 Travis WD. Vasculitis. In: Tomasheski JF Jr, Cagle PT, Farver CF, et al., eds. Dail and Hammar's Pulmonary Pathology. Vol I Nonneoplastic lung disease. 3rd Edn. New York, Springer, 2008; p. 1105.

7 Fukushima Y, Kamiya K, Tatewaki M, et al. A patient with bronchial asthma in whom eosinophilic bronchitis and bronchiolitis developed during treatment. Allergol Int 2010; 59: 87-91.

8 Wenzel SE, Vitari CA, Shende M, et al. Asthmatic granulomatosis: a novel disease with asthmatic and granulomatous changes. Am J Respir Crit Care Med 2012; 186: 501-507. 